\title{
Acute eosinophilic myocarditis as debut of a vasculitis
}

\author{
Miocarditis aguda eosinofílica como presentación inicial de una vasculitis \\ Alejandro Junco-Vicente ${ }^{1 *}$, Luis Caminal-Montero², and José Rozado-Castaño ${ }^{1}$ \\ ${ }^{1}$ Department of Cardiology; ${ }^{2}$ Internal Medicine Systemic Autoimmune Diseases Unit. Hospital Universitario de Asturias, Oviedo, Spain
}

Forty-seven-year-old woman with a history of asthma, who presented with central chest pleural-pericardial pain in cardiogenic shock situation with elevated markers of myocardial damage and moderate systolic dysfunction due to global left ventricular hypokinesia, with moderate concentric hypertrophy and no segmental contractility alterations found on echocardiogram. After receiving hemodynamic support, stabilization was achieved with coronary artery disease being ruled out by coronary artery computed tomography. In the next few days, she showed improvement of the acute heart failure condition, but a marked general syndrome persisted. Serology testing for viruses was negative. Marked and growing peripheral eosinophilia and anti-neutrophil-myeloperoxidase-positive cytoplasmic antibodies were detected. With myocarditis being suspected, cardiac magnetic resonance imaging (MRI) was performed (Fig. 1A-F), which confirmed the acute myocarditis diagnosis. In view of the findings and with clinical suspicion of a systemic condition, an endomyocardial biopsy (EMB) was performed, which demonstrated marked eosinophil infiltration (Fig. 2A and B). All this confirmed the acute eosinophilic myocarditis diagnosis as initial presentation of systemic vasculitis of the eosinophilic granulomatosis with polyangiitis type (formerly Churg-Strauss syndrome). High-dose corticosteroid intravenous treatment was implemented with complete improvement of the patient, subsequently continuing with maintenance doses at home. At third-month control visit, she was asymptomatic with eosinophilia normalization in peripheral blood, and control cardiac MRI showed the resolution of the condition without sequelae (Fig. 1C-H). Myocarditis is a serious entity, mostly being viral of origin, but there are other less common etiologies that should not be forgotten within the differential diagnosis. We highlight eosinophilic myocarditis, which requires histological confirmation by EMB. Its clinical presentation is variable, from minimally symptomatic cases to fulminant forms (called necrotizing eosinophilic myocarditis). In general, it has heart failure and acute coronary syndrome associated symptoms, since eosinophils generate acute cardiomyocyte necrosis. It is an extremely uncommon entity that is associated with high mortality, with early diagnosis and treatment being decisive for improving its prognosis.

\section{Funding}

None.

\section{Conflicts of interest}

The authors declare that they have no conflicts of interest.

\section{Correspondence:}

*Alejandro Junco-Vicente

E-mail: ajuncovicente@gmail.com
Date of reception: 11-12-2019

Date of acceptance: 25-05-2020

DOI: 10.24875/ACME.M21000181
Available online: 25-05-2021

Arch Cardiol Mex (Eng). 2021;91(1):110-111 www.archivoscardiologia.com
article under the CC BY-NC-ND license (http://creativecommons.org/licenses/by-nc-nd/4.0/). 


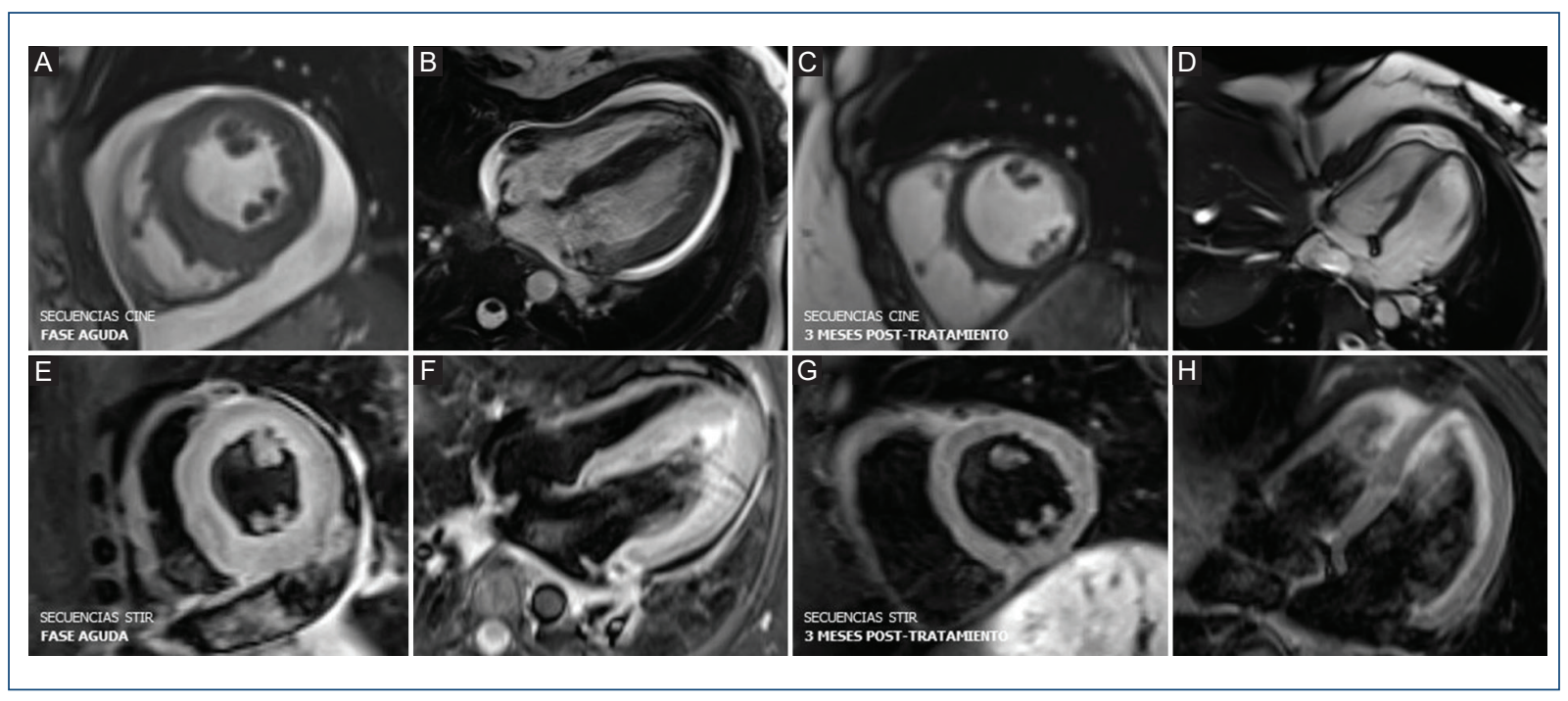

Figure 1. Cardiac magnetic resonance imaging. Baseline study in the acute phase of clinical presentation, where CINE sequences (A: Short axis at mid-ventricular level and B: 4-chamber) show concentric ventricular hypertrophy, as well as circumferential pericardial effusion. In T2-weighted sequences (STIR); C and D: CINE sequences with relevant improvement, decreased concentric ventricular hypertrophy with persistence of mild circumferential pericardial effusion; E and F: Marked hyperintensity is observed (T2 ratio > 2), suggestive of diffuse myocardial edema. At 3 months of steroid treatment; $\mathbf{G}$ and $\mathbf{H}$ : Myocardial edema also being improved, without hyperintensity in T2-weighted sequences being observed.

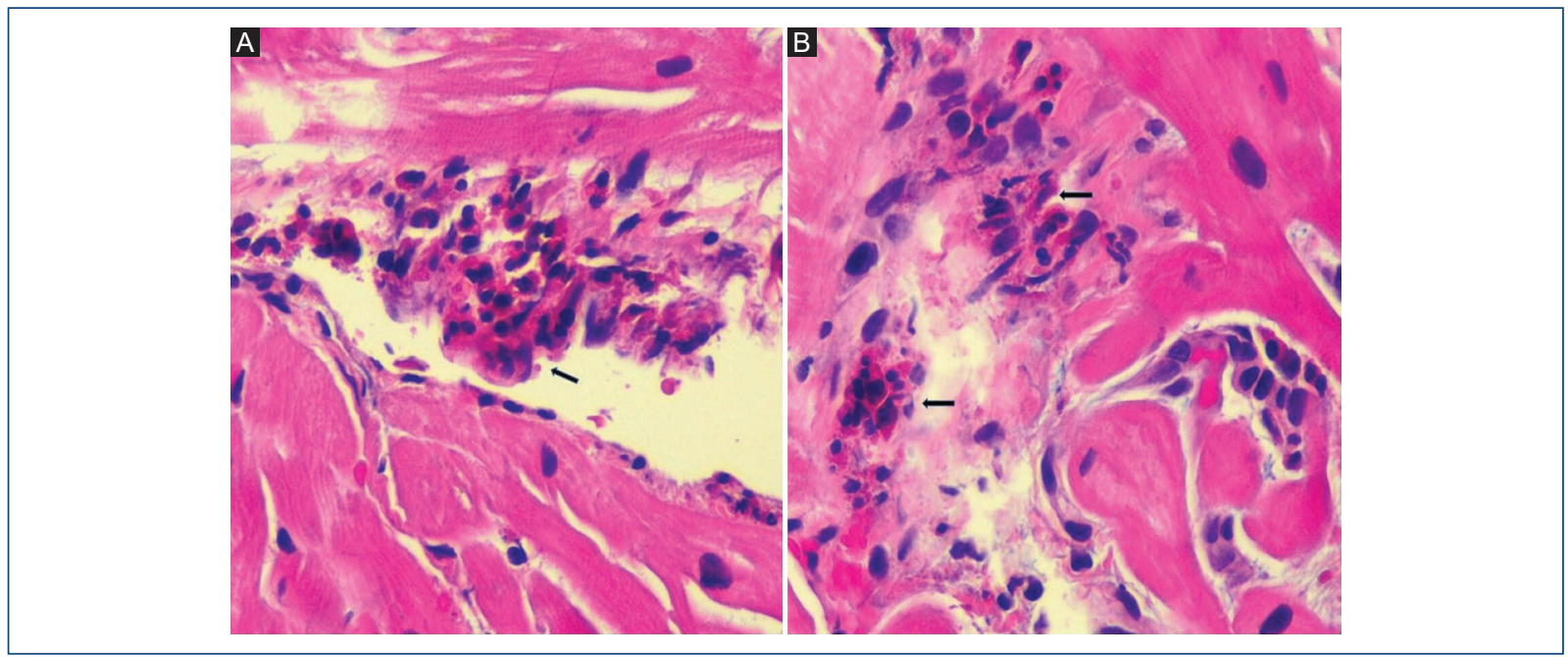

Figure 2. Endomyocardial biopsy light microscopy histological images ( $\times 40$ magnification) with hematoxylin-eosin staining. A: Marked eosinophil infiltration with interstitial edema (black arrows) is observed at the perivascular level and B: Between the myocardial fibers.

\section{Ethical disclosures}

Protection of human and animal subjects. The authors declare that no experiments were performed on humans or animals for this research.
Confidentiality of data. The authors declare that no patient data appear in this article.

Right to privacy and informed consent. The authors declare that no patient data appear in this article. 